\title{
Synthesis and evaluation of new benzoxazole derivatives as potential antiglioma agents
}

\author{
Mehlika Dilek ALTINTOP 1*, Gülşen AKALIN ÇİFTÇİ 2, Halide Edip TEMEL ${ }^{2}$
}

1 Department of Pharmaceutical Chemistry, Faculty of Pharmacy, Anadolu University, 26470 Eskişehir, Turkey.

2 Department of Biochemistry, Faculty of Pharmacy, Anadolu University, 26470 Eskişehir, Turkey.

* Corresponding Author. E-mail: mdaltintop@anadolu.edu.tr (M.D.A.); Tel. +90-222-335 05 80/3772; Fax. +90-222-335 07 50; ORCID No: 0000-0002-8159-663X.

Received: 29 December 2017 / Revised: 05 March 2018 / Accepted: 11 March 2018

\begin{abstract}
Gliomas account for the majority of human brain tumors and the incidence of gliomas is expected to rise in upcoming years and therefore extensive efforts have been devoted to the discovery of potent antiglioma agents. Due to the importance of benzoxazoles for anticancer drug discovery, herein new benzoxazole-based hydrazone derivatives (3a-g) were designed and synthesized. The cytotoxic effects of the compounds on C6 rat glioma and NIH/3T3 mouse embryonic fibroblast cell lines were investigated using MTT assay. The apoptotic effects of the most selective anticancer agent were analyzed based on Annexin V-PI binding capacities in flow cytometry. The compounds were also investigated for their acetylcholinesterase (AChE) inhibitory effects using a modification of Ellman's spectrophotometric method. In order to evaluate the compliance of the compounds to Lipinski's rule of five, their physicochemical parameters were determined using Molinspiration software. $N^{\prime}-\left(\left[1,1^{\prime}\right.\right.$-Biphenyl]-4-ylmethylene)-2-[(5fluorobenzoxazol-2-yl)thio]acetohydrazide $(3 \mathrm{~g})$ was found to be more effective on C6 cell line $\left(\mathrm{IC}_{50}=4.30 \pm 0.28 \mu \mathrm{g} / \mathrm{mL}\right)$ than mitoxantrone $\left(\mathrm{IC}_{50}=4.56 \pm 1.24 \mu \mathrm{g} / \mathrm{mL}\right)$. The high SI value of compound $3 \mathrm{~g}$ indicated that its antiglioma activity was selective. This compound caused late apoptosis in a dose dependent manner. Compound $3 \mathbf{g}$ was also found to be the most effective AChE inhibitor in this series. According to in silico studies, compound $\mathbf{3 g}$ only violated one parameter of Lipinski's rule of five. On the basis of Lipinski's rule, the compound was expected to have reasonable oral bioavailability. According to in vitro and in silico studies, compound $\mathbf{3 g}$ stands out as a promising antiglioma agent for further studies.
\end{abstract}

KEYWORDS: Acetylcholinesterase; apoptosis; benzoxazole; glioma; hydrazone.

\section{INTRODUCTION}

Gliomas are the most common primary malignant brain tumors and the incidence of gliomas is expected to rise in upcoming years. A common approach for the treatment of glioma involves surgery, radiotherapy and chemotherapy. However, there is no $100 \%$ curative therapy available for glioma patients and survival rates related to the most malignant types of gliomas are very low. Recurrences are also frequent since conventional therapies do not take into account the unique molecular features of different subtypes of glioma [1-3].

Efficacy of chemotherapy is limited due to poor drug delivery to the brain tumor and the correspondingly limited therapeutic response caused by partially intact blood-brain barrier (BBB) and bloodtumor barrier (BTB). Efficacy of anticancer agents is also reduced by the inherent chemoresistance of brain endothelial and glioma cells expressing the drug efflux protein, P-glycoprotein. As a result, extensive efforts have been devoted to the discovery of new antiglioma agents to improve drug delivery across the BBB/BTB and overcome drug resistance [1-3].

Benzoxazoles represent an important class of chemical entities in medicinal chemistry as building blocks for modulating a ligand's affinity and/or selectivity towards a particular target and therefore benzoxazole scaffold is often found in ligands targeting a plethora of receptors and enzymes. Benzoxazole derivatives have been reported to show a wide range of biological activities such as antibacterial, antifungal, antiviral, anticancer, and antioxidant activities [4-6]. In particular, recent studies have indicated that benzoxazole derivatives show potent antitumor activity against different cancer cell lines [7-12] through the inhibition of

How to cite this article: Altintop MD, Akalin Ciftci G, Temel HE. Synthesis and evaluation of new benzoxazole derivatives as potential antiglioma agents. Marmara Pharm J. 2018; 22 (4): 547-558. 
Aurora B kinase [8], cyclooxygenase-2 (COX-2) [9,10], topoisomerase II [11] and the induction of Caspase-3 dependent apoptosis [12].

Hydrazides-hydrazones have also attracted a great deal of interest as an important class of lead compounds for the development of new chemical entities to treat various diseases due to their unique structural features and diverse biological activities [13-15]. In particular, hydrazone moiety plays a pivotal role in anticancer drug development. Hydrazone derivatives have been reported to show potent antitumor activity against various cancer cell lines such as U-373 MG human glioblastoma, MCF-7 human breast adenocarcinoma, A549 human lung carcinoma, SK-OV-3 human ovary carcinoma, SK-MEL-2 human melanoma, HCT15 human colon carcinoma, MIA PaCa-2 human pancreas carcinoma, and HepG2 human hepatocellular carcinoma cell lines [14-20].

Prompted by the aforementioned findings, herein we designed, synthesized new benzoxazole-based hydrazone derivatives and tested their in vitro cytotoxic effects on C6 rat glioma and NIH/3T3 mouse embryonic fibroblast cell lines followed by flow cytometry-based apoptosis detection in C6 cell line. The compounds were also investigated for their acetylcholinesterase (AChE) inhibitory effects. A computational study for the prediction of absorption, distribution, metabolism and excretion (ADME) properties of all compounds was performed.

\section{RESULTS AND DISCUSSION}

The synthesis of new hydrazone derivatives (3a-g) was carried out according to the steps shown in Figure 1. In the initial step, ethyl 2-[(5-fluorobenzoxazol-2-yl)thio]acetate (1) was synthesized via the reaction of 5-fluorobenzoxazole-2-thiol with ethyl chloroacetate in the presence of potassium carbonate. The treatment of the ester (1) with hydrazine hydrate afforded the corresponding hydrazide (2). Finally, the nucleophilic addition-elimination reaction of the hydrazide (2) with aromatic aldehydes gave the target compounds (3a-g).<smiles>CC#CCCOC(=O)CSc1nc2cc(F)ccc2o1</smiles>

1<smiles>NNC(=O)CSc1nc2cc(F)ccc2o1</smiles>

2

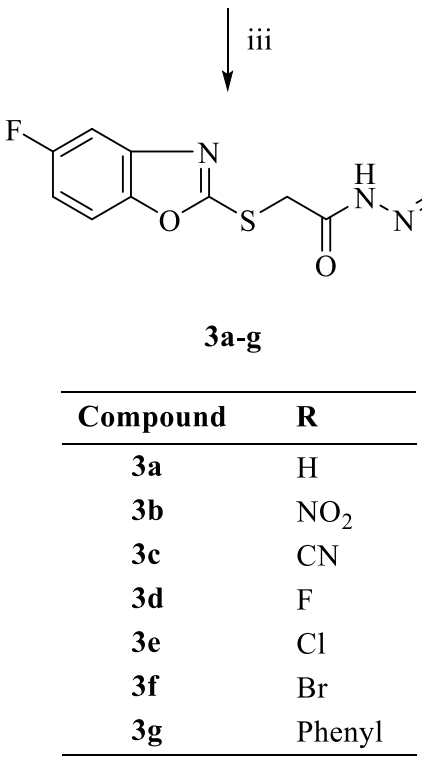

Figure 1. The synthetic route for the preparation of compounds 3a-g. Reagents and conditions: (i) $\mathrm{ClCH}_{2} \mathrm{COOEt}, \mathrm{K}_{2} \mathrm{CO}_{3}$, acetone, reflux, 10 h; (ii) $\mathrm{NH}_{2} \mathrm{NH}_{2} \cdot \mathrm{H}_{2} \mathrm{O}$, ethanol, rt, $3 \mathrm{~h}$; (iii) Aromatic aldehyde, ethanol, reflux, $6 \mathrm{~h}$.

The structures of the compounds were confirmed by IR, ${ }^{1} \mathrm{H}$ NMR, ${ }^{13} \mathrm{C}$ NMR, mass spectral data, and elemental analysis. In the IR spectrum of compound 2 , the $\mathrm{C}=\mathrm{O}$ stretching vibrations gave rise to a band at $1641.42 \mathrm{~cm}^{-1}$. The N-H stretching bands were observed in the region 3298-3203 $\mathrm{cm}^{-1}$. The stretching bands for aromatic and aliphatic C-H groups occurred at 3111-3043 $\mathrm{cm}^{-1}$ and $2995-2939 \mathrm{~cm}^{-1}$, respectively. N-H bending, 
$\mathrm{C}=\mathrm{N}, \mathrm{C}=\mathrm{C}$ stretching bands were observed in the region $1625-1462 \mathrm{~cm}^{-1}$. In the IR spectra of compounds 3a-g, a strong, characteristic band in the region $1687-1658 \mathrm{~cm}^{-1}$ due to the $\mathrm{C}=\mathrm{O}$ stretching vibration was observed. The N-H stretching bands occurred at $3253-3184 \mathrm{~cm}^{-1}$. The aromatic and aliphatic $\mathrm{C}-\mathrm{H}$ stretching vibrations gave rise to bands at $3142-3030 \mathrm{~cm}^{-1}$ and $2980-2846 \mathrm{~cm}^{-1}$, respectively. $\mathrm{C}=\mathrm{N}, \mathrm{C}=\mathrm{C}$ stretching and $\mathrm{N}-\mathrm{H}$ bending vibrations were observed in the region 1616-1463 $\mathrm{cm}^{-1}$. In the IR spectrum of cyano-substituted compound 3c, the $\mathrm{C} \equiv \mathrm{N}$ stretching band occurred at $2229.71 \mathrm{~cm}^{-1}$.

In the ${ }^{1} \mathrm{H}$ NMR spectrum of compound 2 , the signals due to the $\mathrm{NH}_{2}$ and $\mathrm{NH}$ protons of the hydrazide group appeared at 4.35 and $9.43 \mathrm{ppm}$, respectively. The signal due to the $\mathrm{S}-\mathrm{CH}_{2}$ protons was observed at 4.08 ppm as a singlet. In the ${ }^{1} \mathrm{H}$ NMR spectra of compounds 3a-g, the signal due to the hydrazone proton appeared in the region 11-12 ppm as a singlet. The signals due to the $\mathrm{S}-\mathrm{CH}_{2}$ protons were observed in the region 4.28$4.73 \mathrm{ppm}$. The signal belonging to $\mathrm{CH}=\mathrm{N}$ proton appeared in the region 8.01-8.33 ppm. In the ${ }^{1} \mathrm{H}$ NMR spectra of compounds 3a-g, $\mathrm{CH}=\mathrm{N}$ and $\mathrm{S}-\mathrm{CH}_{2}$ protons gave rise to two singlet peaks in accordance with the presence of the $E$ and $Z$ isomers [21,22].

In the ${ }^{13} \mathrm{C}$ NMR spectra of all compounds, the signal due to the $\mathrm{S}-\mathrm{CH}_{2}$ carbon was observed in the region $34-35 \mathrm{ppm}$. The signal due to the $\mathrm{C}=\mathrm{O}$ carbon was observed at $165-169 \mathrm{ppm}$. In the ${ }^{13} \mathrm{C}$ NMR spectra of compounds 3a-g, the signal due to the hydrazone carbon was observed at $144-147 \mathrm{ppm}$. In the ${ }^{13} \mathrm{C}$ NMR spectrum of cyano-substituted compound 3c, the signal due to the $C \equiv N$ carbon appeared at $119.10 \mathrm{ppm}$. HRMS spectral data and elemental analysis were in agreement with the proposed structures of the compounds.

MTT assay was carried out to determine the anticancer effects of the compounds on C6 rat glioma cell line (Table 1). Biphenyl-substituted compound $3 \mathbf{g}$ was found to be the most potent anticancer agent against C6 cell line with an $\mathrm{IC}_{50}$ value of $4.30 \pm 0.28 \mu \mathrm{g} / \mathrm{mL}$ when compared with mitoxantrone $\left(\mathrm{IC}_{50}=4.56 \pm 1.24\right.$ $\mu \mathrm{g} / \mathrm{mL}$ ). This outcome indicated that biphenyl group significantly enhanced anticancer activity against C6 cell line. The increased anticancer activity can be attributed to the increased lipophilicity of the compound due to the presence of biphenyl moiety.

Toxicity to host cells is an important characteristic to assess the safety of drug candidates early in the drug discovery process. The cytotoxic effects of the compounds on NIH/3T3 mouse embryonic fibroblast cell line were investigated to evaluate whether the compounds were toxic or non-toxic to healthy cells. The selectivity index (SI) values of the compounds were also determined to compare the selectivity of the compounds (Table 1). The high SI value of compound $3 g$ pointed out that the antiglioma activity of compound $\mathbf{3 g}$ was selective. Due to its notable and selective antitumor effect on C6 cells, compound $\mathbf{3} \mathbf{g}$ was chosen for further studies.

Table 1. $\mathrm{IC}_{50}$ values of the compounds against $\mathrm{C} 6$ and NIH/3T3 cells for $24 \mathrm{~h}$

\begin{tabular}{|c|c|c|c|}
\hline \multirow{2}{*}{ Compound } & \multicolumn{2}{|c|}{$\mathrm{IC}_{50}$ values $(\mu \mathrm{g} / \mathrm{mL})$} & \multirow{2}{*}{ SI value* } \\
\hline & C6 cell line & NIH/3T3 cell line & \\
\hline 2 & $>500$ & $>500$ & - \\
\hline $3 a$ & $>500$ & $>500$ & - \\
\hline $3 b$ & $46.00 \pm 6.92$ & $51.67 \pm 2.89$ & 1.12 \\
\hline $3 c$ & $>500$ & $>500$ & - \\
\hline $3 d$ & $>500$ & $>500$ & - \\
\hline $3 e$ & $>500$ & $>500$ & - \\
\hline $3 f$ & $>500$ & $>500$ & - \\
\hline $3 g$ & $4.30 \pm 0.28$ & $>500$ & $>116.28$ \\
\hline Mitoxantrone & $4.56 \pm 1.24$ & ND & - \\
\hline
\end{tabular}

ND: Not determined.

* $\mathrm{SI}=\mathrm{IC}_{50}$ for normal cell line $/ \mathrm{IC}_{50}$ for cancerous cell line.

After $24 \mathrm{~h}$ incubation period, the apoptotic effects of compound $\mathbf{3 g}$ were analyzed based on Annexin VPI binding capacities in flow cytometry (Table 2, Figure 2). Following flow cytometric analyses, the early and late apoptotic effects of compound $\mathbf{3 g}$ (for $\mathrm{IC}_{50} / 2$ and $\mathrm{IC}_{50}$ doses) on $\mathrm{C} 6$ cell line were determined as $9.3 \%$ and $8.7 \%$, respectively. According to these findings, the early apoptotic effects of this compound did not increase in a dose dependent manner, whereas the late apoptotic effects increased in a dose dependent manner. 
Table 2. Percents of typical quadrant analysis of Annexin V FITC/Propidium iodide (PI) flow cytometry of C6 cells treated with compound $3 \mathrm{~g}$ and mitoxantrone at $\mathrm{IC}_{50} / 2$ and $\mathrm{IC}_{50}$ concentrations

\begin{tabular}{cccc}
\hline Groups & Early apoptotic cells\% & Late apoptotic cells\% & Viable cells\% \\
\hline Control (untreated) & 2.5 & 3.2 & 92.8 \\
Compound 3g IC $50 / 2$ dose treated cells & 2.7 & 6.6 & 70.9 \\
Compound 3g IC 50 dose treated cells & 0.8 & 7.9 & 87.3 \\
Mitoxantrone IC 50 /2 dose treated cells & 2.9 & 7.9 & 43.4 \\
Mitoxantrone IC 50 dose treated cells & 1.6 & 8.0 & 40.5 \\
\hline
\end{tabular}

C6 cells were cultured for $24 \mathrm{~h}$ in medium with compound $3 \mathrm{~g}$ and mitoxantrone at $\mathrm{IC}_{50} / 2$ and $\mathrm{IC}_{50}$ concentrations. At least 10.000 cells were analyzed per sample, and quadrant analysis was performed.

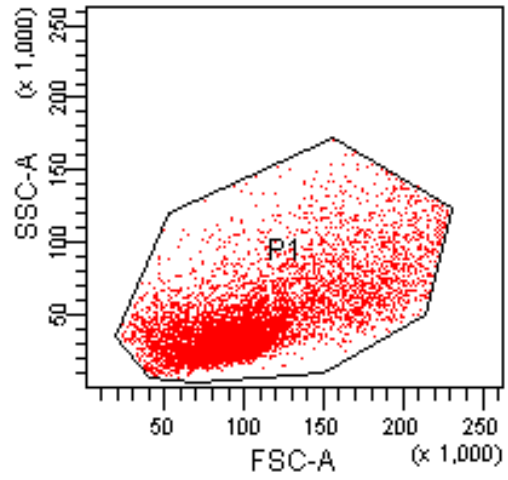

Control

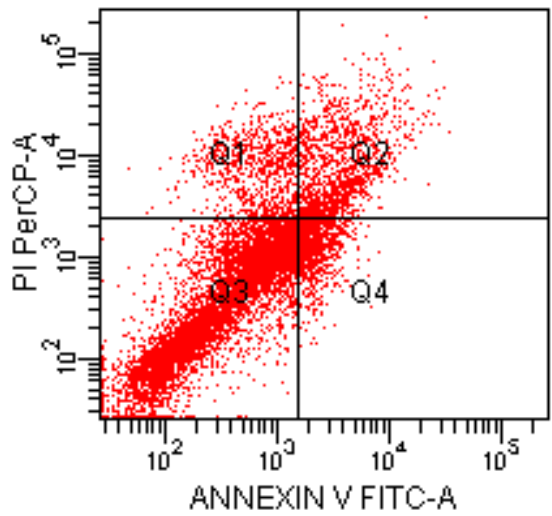

Compound $3 \mathrm{~g}$ at $\mathrm{IC}_{50} / 2$

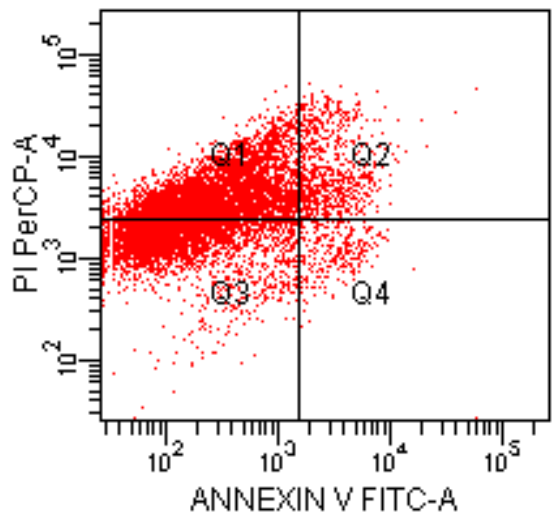

Mitoxantrone $\mathrm{IC}_{50} / 2$

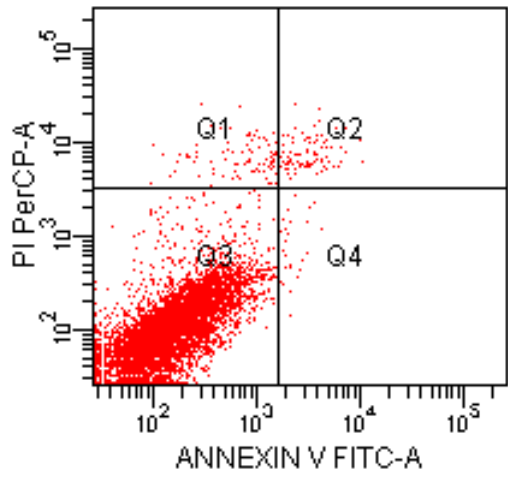

Control

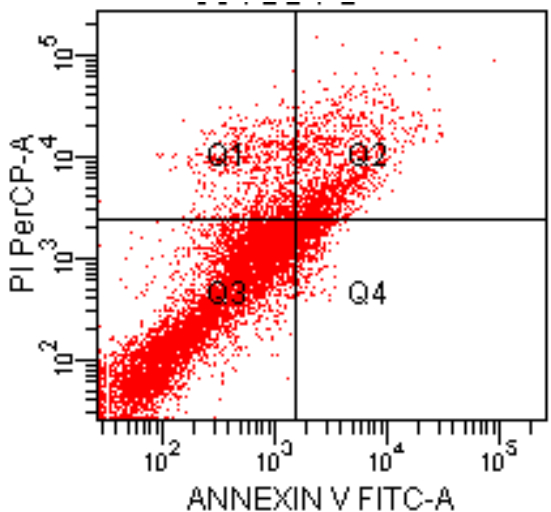

Compound $3 g$ at $\mathrm{IC}_{50}$

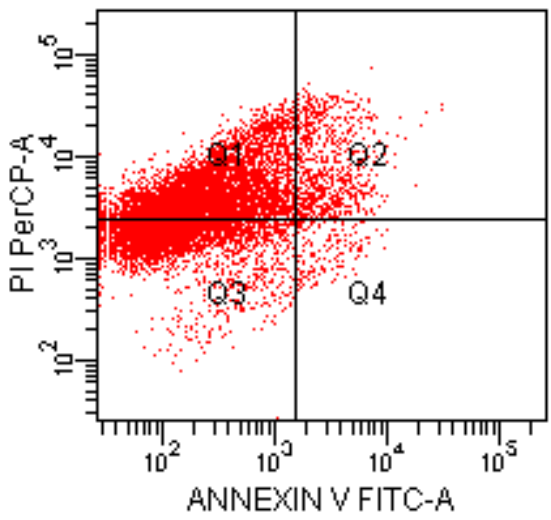

Mitoxantrone $\mathrm{IC}_{50}$

Figure 2. Flow cytometric analysis of $\mathrm{C} 6$ cells treated with compound $3 \mathrm{~g}$ and mitoxantrone at $\mathrm{IC}_{50}$ and $\mathrm{IC}_{50} / 2$ concentrations. C6 cells were cultured for $24 \mathrm{~h}$ in medium with compound $3 \mathrm{~g}$ and mitoxantrone at $\mathrm{IC}_{50} / 2$ and $\mathrm{IC}_{50}$ concentrations. At least 10,000 cells were analyzed per sample, and quadrant analysis was performed. 
The inhibitory effects of the compounds on AChE were also determined by a modification of Ellman's spectrophotometric method (Table 3). Galantamine was used as a positive control. According to the results, compounds 3a-g showed less AChE inhibitory activity than galantamine. Compounds $\mathbf{2}, \mathbf{3 a}, \mathbf{3 b}, \mathbf{3 c}, \mathbf{3 d}, \mathbf{3} \mathbf{f}$ and $3 \mathrm{~g}$ showed less than $50 \% \mathrm{AChE}$ inhibition at $80 \mu \mathrm{g} / \mathrm{mL}$, whereas compound 3e did not show any inhibitory activity against $\mathrm{AChE}$. Compound $3 \mathrm{~g}$ was identified as the most potent $\mathrm{AChE}$ inhibitor $(45.98 \pm 3.13 \%)$ in this series. This outcome indicated that biphenyl moiety enhanced AChE inhibitory activity. The increased activity can be attributed to its high lipophilicity due to the presence of biphenyl moiety.

Table 3. The inhibitory effects of the compounds on AChE

\begin{tabular}{cc}
\hline Compound & AChE\% inhibition $(\mathbf{8 0} \boldsymbol{\mu g} / \mathbf{m L})$ \\
\hline $\mathbf{2}$ & $19.77 \pm 2.29$ \\
$\mathbf{3 a}$ & $27.41 \pm 1.88$ \\
$\mathbf{3 b}$ & $28.48 \pm 2.71$ \\
$\mathbf{3 c}$ & $18.08 \pm 3.06$ \\
$\mathbf{3 d}$ & $19.93 \pm 3.20$ \\
$\mathbf{3 e}$ & --- \\
3f & $10.91 \pm 0.76$ \\
$\mathbf{3 g}$ & $45.98 \pm 3.13$ \\
\hline Galantamine & $(0.45 \pm 0.04)^{\mathrm{a}}$ \\
\hline$---:$ No inhibition & \\
a IC 50 value $(\mu \mathrm{g} / \mathrm{mL})$ &
\end{tabular}

In order to evaluate the compliance of the compounds to Lipinski's rule of five, Molinspiration software was used to determine their physicochemical parameters (log P, TPSA, nrotb, molecular weight, number of hydrogen bond donors and acceptors, molecular volume) [23]. According to Lipinski's rule of five, most "drug like" molecules have $\log \mathrm{P} \leq 5$, molecular weight $\leq 500$, number of hydrogen bond acceptors $\leq 10$, and number of hydrogen bond donors $\leq 5$ and compounds violating more than one of these rules may have bioavailability problems [23-26]. According to in silico studies, compound $3 \mathrm{~g}$ only violated one parameter of Lipinski's rule of five, whereas other compounds did not violate Lipinski's rule (Table 4). On the basis of Lipinski's rule of five, they were expected to have good oral bioavailability.

Table 4. The predicted pharmacokinetic parameters of the compounds

\begin{tabular}{ccccccccc}
\hline \multirow{2}{*}{ Compound } & \multicolumn{7}{c}{ Molecular properties a } \\
\cline { 2 - 9 } & MW & logP & TPSA & nrotb & HBA & HBD & Volume & Violations \\
\hline $\mathbf{2}$ & 241.25 & 0.25 & 81.15 & 3 & 5 & 3 & 187.98 & 0 \\
3a & 329.36 & 3.66 & 67.49 & 5 & 5 & 1 & 271.36 & 0 \\
3b & 374.35 & 3.62 & 113.32 & 6 & 8 & 1 & 294.70 & 0 \\
3c & 354.37 & 3.42 & 91.29 & 5 & 6 & 1 & 288.22 & 0 \\
3d & 347.35 & 3.83 & 67.49 & 5 & 5 & 1 & 276.30 & 0 \\
3e & 363.80 & 4.34 & 67.49 & 5 & 5 & 1 & 284.90 & 0 \\
3f & 408.25 & 4.47 & 67.49 & 5 & 5 & 1 & 289.25 & 0 \\
3g & 405.45 & 5.46 & 67.49 & 6 & 5 & 1 & 342.77 & 1 \\
\hline
\end{tabular}

a Molecular properties were calculated using Molinspiration software. MW: Molecular weight; logP: The logarithm of octanol/water partition coefficient, TPSA: Topological polar surface area; nrotb: Number of rotatable bonds, HBA: Number of hydrogen bond acceptors, HBD: Number of hydrogen bond donors.

\section{CONCLUSION}

In the present paper, new benzoxazole-based hydrazone derivatives were synthesized and investigated for their cytotoxic effects on C6 rat glioma, and NIH/3T3 mouse embryonic fibroblast cell lines and AChE inhibitory effects. Moreover, the compliance of the compounds to the Lipinski's rule of five was evaluated.

Biphenyl-substituted compound $\mathbf{3 g}$ was identified as the most promising anticancer agent against $\mathrm{C6}$ cell line due to its selective and notable anticancer activity $\left(\mathrm{IC}_{50}=4.30 \pm 0.28 \mu \mathrm{g} / \mathrm{mL}\right)$ when compared with mitoxantrone $\left(\mathrm{IC}_{50}=4.56 \pm 1.24 \mu \mathrm{g} / \mathrm{mL}\right)$. The apoptotic effects of compound $3 \mathrm{~g}$ were also investigated. This compound caused late apoptosis in a dose dependent manner. According to the in vitro and in silico studies, compound $3 \mathrm{~g}$ stands out as a promising orally bioavailable anticancer drug candidate for further in vitro and in vivo studies. 


\section{MATERIALS AND METHODS}

\subsection{Chemistry}

All reagents were purchased from commercial suppliers and were used without further purification. The melting points (M.p.) of the compounds were determined on a MP90 digital melting point apparatus (Mettler Toledo, Ohio, USA) and are uncorrected. IR spectra were recorded on an IRPrestige-21 Fourier Transform Infrared spectrophotometer (Shimadzu, Tokyo, Japan). ${ }^{1} \mathrm{H}$ NMR and ${ }^{13} \mathrm{C}$ NMR spectra were recorded on a Bruker spectrometer (Bruker, Billerica, MA, USA). Chemical shifts were reported in parts per million (ppm) and the coupling constants $(J)$ were expressed in Hertz $(\mathrm{Hz})$. Mass spectra were recorded on a Shimadzu LCMS-IT-TOF system (Shimadzu, Kyoto, Japan). Elemental analyses (C, H, N) were performed on a Perkin Elmer EAL 240 elemental analyzer (Perkin-Elmer, Norwalk, CT, USA). Thin Layer Chromatography (TLC) was performed on TLC Silica gel 60 F254 aluminium sheets (Merck, Darmstadt, Germany) to check the purity of the compounds.

General procedure for the synthesis of the compounds

Ethyl 2-[(5-fluorobenzoxazol-2-yl)thio]acetate (1)

A mixture of 5-fluorobenzoxazole-2-thiol $(0.05 \mathrm{~mol})$ and ethyl chloroacetate $(0.05 \mathrm{~mol})$ in the presence of potassium carbonate $(0.05 \mathrm{~mol})$ in acetone was refluxed for $10 \mathrm{~h}$. The reaction mixture was cooled, filtered and the crude product was solved in water and then extracted with ether [22].

\section{2-[(5-Fluorobenzoxazol-2-yl)thio]acetohydrazide (2)}

A mixture of the ester $(\mathbf{1})(0.05 \mathrm{~mol})$ and hydrazine hydrate $(0.1 \mathrm{~mol})$ in ethanol was stirred at room temperature for $3 \mathrm{~h}$ and then filtered [22].

M.p. $174.6^{\circ} \mathrm{C}$. Yield $88 \%$.

IR $v_{\max }\left(\mathrm{cm}^{-1}\right)$ : 3298.28, 3203.76 (N-H stretching), 3111.18, 3089.96, 3043.67 (Aromatic C-H stretching), 2995.45, 2939.52 (Aliphatic C-H stretching), 1641.42 (C=O stretching), 1625.99, 1537.27, 1494.83, 1473.62, 1462.04 (N-H bending, $\mathrm{C}=\mathrm{N}$ and $\mathrm{C}=\mathrm{C}$ stretching), 1435.04, 1408.04 ( $\mathrm{C}-\mathrm{H}$ bending), 1338.60, 1269.16, 1251.80, 1238.30, 1217.08, 1178.51, 1139.93, 1128.36, 1001.06 (C-N, C-O stretching and aromatic C-H in plane bending), 956.69, 935.48, 854.47, 831.32, 804.32, 771.53, 727.16, 678.94 (Aromatic C-H out of plane bending and C-S stretching).

${ }^{1} \mathrm{H}$ NMR (300 MHz, DMSO- $\left.d_{6}\right): 4.08$ (s, 2H, S- $\left.\mathrm{CH}_{2}\right), 4.35\left(\mathrm{~s}, 2 \mathrm{H}, \mathrm{NH}_{2}\right), 7.13-7.20(\mathrm{~m}, 1 \mathrm{H}$, benzoxazole), 7.50 (dd, $J=2.61 \mathrm{~Hz}, J=8.76 \mathrm{~Hz}, 1 \mathrm{H}$, benzoxazole), $7.66(\mathrm{dd}, J=4.35 \mathrm{~Hz}, J=8.91 \mathrm{~Hz}, 1 \mathrm{H}$, benzoxazole), $9.43(\mathrm{~s}, 1 \mathrm{H}, \mathrm{N}-\mathrm{H})$.

${ }^{13} \mathrm{C}$ NMR (75 MHz, DMSO- $\left.d_{6}\right): 34.43\left(\mathrm{CH}_{2}, \mathrm{~S}-\mathrm{CH}_{2}\right), 105.42(\mathrm{CH}, \mathrm{d}, \mathrm{J}=26.25 \mathrm{~Hz}$, benzoxazole), $111.37(\mathrm{CH}, \mathrm{d}, \mathrm{J}=$ $10.5 \mathrm{~Hz}$, benzoxazole), $111.92(\mathrm{CH}, \mathrm{d}, \mathrm{J}=26.25 \mathrm{~Hz}$, benzoxazole), $142.58(\mathrm{C}, \mathrm{d}, \mathrm{J}=13.5 \mathrm{~Hz}$, benzoxazole), 148.27 (C, benzoxazole), 158.32 ( $C$, benzoxazole), 161.47 (C, benzoxazole), 165.96 (C, C=O).

HRMS (ESI) $(m / z):[\mathrm{M}+\mathrm{H}]^{+}$calcd. for $\mathrm{C}_{9} \mathrm{H}_{8} \mathrm{FN}_{3} \mathrm{O}_{2} \mathrm{~S}: 242.0394$, found: 242.0387 .

For $\mathrm{C}_{9} \mathrm{H}_{8} \mathrm{FN}_{3} \mathrm{O}_{2} \mathrm{~S}$ Calculated: $\mathrm{C}, 44.81 ; \mathrm{H}, 3.34 ; \mathrm{N}, 17.42$. Found: $\mathrm{C}, 44.78 ; \mathrm{H}, 3.36 ; \mathrm{N}, 17.43$.

N'-Benzylidene-2-[(5-fluorobenzoxazol-2-yl)thio]acetohydrazide derivatives (3a-g)

A mixture of the hydrazide (2) $(0.01 \mathrm{~mol})$ and appropriate aldehyde $(0.01 \mathrm{~mol})$ was refluxed in ethanol for $6 \mathrm{~h}$, filtered and crystallized from ethanol [22].

N'-Benzylidene-2-[(5-fluorobenzoxazol-2-yl)thio]acetohydrazide (3a)

M.p. $190.5^{\circ} \mathrm{C}$. Yield $82 \%$. 
IR $v_{\max }\left(\mathrm{cm}^{-1}\right)$ : 3184.48 (N-H stretching), 3099.61 (Aromatic C-H stretching), 2976.16, 2870.08 (Aliphatic C-H stretching), 1672.28 ( $\mathrm{C}=\mathrm{O}$ stretching), 1614.42, 1490.97, 1473.62, $1463.97(\mathrm{~N}-\mathrm{H}$ bending, $\mathrm{C}=\mathrm{N}$ and $\mathrm{C}=\mathrm{C}$ stretching), 1448.54, 1435.04, 1388.75, 1367.53, 1352.10 (C-H bending), 1315.45, 1234.44, 1203.58, 1130.29, 1074.35 (C-N, C-O stretching and aromatic C-H in plane bending), 952.84, 927.76, 898.83, 885.33, 860.25, 829.39, 798.53, 783.10, 750.31, 690.52 (Aromatic C-H out of plane bending and C-S stretching).

${ }^{1} \mathrm{H}$ NMR (300 MHz, DMSO- $\left.d_{6}\right): 4.29$ and $4.69\left(2 \mathrm{~s}, 2 \mathrm{H}, \mathrm{S}-\mathrm{CH}_{2}\right), 7.16(\mathrm{t}, J=8.46 \mathrm{~Hz}, 1 \mathrm{H}, \mathrm{Ar}-\mathrm{H}), 7.43(\mathrm{~s}, 1 \mathrm{H}, \mathrm{Ar}-\mathrm{H})$, $7.52(\mathrm{~d}, J=8.55 \mathrm{~Hz}, 3 \mathrm{H}, \mathrm{Ar}-\mathrm{H}), 7.69$ (brs, 3H, Ar-H), 8.05 and $8.22(2 \mathrm{~s}, 1 \mathrm{H}, \mathrm{CH}=\mathrm{N}), 11.79(\mathrm{~s}, 1 \mathrm{H}, \mathrm{N}-\mathrm{H})$.

${ }^{13} \mathrm{C}$ NMR (75 MHz, DMSO-d $\left.d_{6}\right): 35.28\left(\mathrm{CH}_{2}, \mathrm{~S}-\mathrm{CH}_{2}\right), 105.47(\mathrm{CH}, \mathrm{d}, J=26.25 \mathrm{~Hz}$, aromatic), 111.33 (CH, d, J= 10.5 $\mathrm{Hz}$, aromatic), $111.85(\mathrm{CH}, \mathrm{d}, J=26.25 \mathrm{~Hz}$, aromatic), $127.51(2 \mathrm{CH}, \mathrm{d}, J=16.5 \mathrm{~Hz}$, aromatic), $129.29(2 \mathrm{CH}$, aromatic), $130.60(\mathrm{CH}, \mathrm{d}, J=10.5 \mathrm{~Hz}$, aromatic), 134.39 (C, d, J=8.25 Hz, aromatic), $142.64(\mathrm{C}, \mathrm{d}, J=12.75 \mathrm{~Hz}$, aromatic), $144.62(\mathrm{CH}, \mathrm{CH}=\mathrm{N}), 147.76(\mathrm{C}$, aromatic), 158.32 (C, aromatic), $161.47(\mathrm{C}$, aromatic), $168.34(\mathrm{C}, \mathrm{C}=\mathrm{O})$.

HRMS (ESI) (m/z): [M+H] $]^{+}$calcd. for $\mathrm{C}_{16} \mathrm{H}_{12} \mathrm{FN}_{3} \mathrm{O}_{2} \mathrm{~S}: 330.0707$, found: 330.0693 .

For $\mathrm{C}_{16} \mathrm{H}_{12} \mathrm{FN}_{3} \mathrm{O}_{2} \mathrm{~S}$ Calculated: $\mathrm{C}, 58.35 ; \mathrm{H}, 3.67 ; \mathrm{N}, 12.76$. Found: $\mathrm{C}, 58.31 ; \mathrm{H}, 3.68 ; \mathrm{N}, 12.79$.

2-[(5-Fluorobenzoxazol-2-yl)thio]-N'-(4-nitrobenzylidene)acetohydrazide (3b)

M.p. $237.3^{\circ} \mathrm{C}$. Yield $91 \%$.

IR $v_{\max }\left(\mathrm{cm}^{-1}\right)$ : 3197.98 (N-H stretching), 3076.46 (Aromatic C-H stretching), 2953.02, 2846.93 (Aliphatic C-H stretching), 1678.07 ( $\mathrm{C}=\mathrm{O}$ stretching), 1614.42, 1585.49, 1519.91, 1485.19, 1469.76 ( $\mathrm{N}-\mathrm{H}$ bending, $\mathrm{NO}_{2}, \mathrm{C}=\mathrm{N}$ and $\mathrm{C}=\mathrm{C}$ stretching), 1404.18, 1381.03 (C-H bending), 1338.60, 1249.87, 1234.44, 1201.65, 1138.00, 1105.21 (C-N, CO stretching and aromatic C-H in plane bending), 952.84, 927.76, 898.83, 875.68, 852.54, 833.25, 806.25, 771.53, 748.38, 690.52 (Aromatic C-H out of plane bending and C-S stretching).

${ }^{1} \mathrm{H}$ NMR (300 MHz, DMSO-d $)$ : 4.32 and $4.73\left(2 \mathrm{~s}, 2 \mathrm{H}, \mathrm{S}-\mathrm{CH}_{2}\right), 7.17(\mathrm{t}, J=9.0 \mathrm{~Hz}, J=9.21 \mathrm{~Hz}, 1 \mathrm{H}, \mathrm{Ar}-\mathrm{H}), 7.53(\mathrm{~d}$, $J=8.61 \mathrm{~Hz}, 1 \mathrm{H}, \mathrm{Ar}-\mathrm{H}), 7.67-7.69(\mathrm{~m}, 1 \mathrm{H}, \mathrm{Ar}-\mathrm{H}), 7.98(\mathrm{~d}, J=7.68 \mathrm{~Hz}, 2 \mathrm{H}, \mathrm{Ar}-\mathrm{H}), 8.15$ and $8.33(\mathrm{~s}, 1 \mathrm{H}, \mathrm{CH}=\mathrm{N})$, $8.28(\mathrm{~d}, J=8.13 \mathrm{~Hz}, 2 \mathrm{H}, \mathrm{Ar}-\mathrm{H}), 12.09(\mathrm{~s}, 1 \mathrm{H}, \mathrm{N}-\mathrm{H})$.

${ }^{13} \mathrm{C}$ NMR (75 MHz, DMSO-d $)$ : $35.14\left(\mathrm{CH}_{2}, \mathrm{~S}-\mathrm{CH}_{2}\right), 105.50(\mathrm{CH}, \mathrm{d}, \mathrm{J}=25.5 \mathrm{~Hz}$, aromatic), 111.39 (CH, d, J= 10.5 $\mathrm{Hz}$, aromatic), $111.92(\mathrm{CH}, \mathrm{d}, \mathrm{J}=26.25 \mathrm{~Hz}$, aromatic), $124.51(2 \mathrm{CH}$, aromatic), $128.38(2 \mathrm{CH}$, aromatic), 140.63 (C, aromatic), 142.27 (C, aromatic), $145.34(\mathrm{CH}, \mathrm{CH}=\mathrm{N}), 148.29$ (C, aromatic), 158.33 (C, aromatic), 161.49 (C, aromatic), 163.72 (C, aromatic), $168.77(\mathrm{C}, \mathrm{C}=\mathrm{O})$.

HRMS (ESI) (m/z): [M+H] $]^{+}$calcd. for $\mathrm{C}_{16} \mathrm{H}_{11} \mathrm{FN}_{4} \mathrm{O}_{4} \mathrm{~S}: 375.0558$, found: 375.0542 .

For $\mathrm{C}_{16} \mathrm{H}_{11} \mathrm{FN}_{4} \mathrm{O}_{4} \mathrm{~S}$ Calculated: C, 51.34; $\mathrm{H}, 2.96 ; \mathrm{N}, 14.97$. Found: $\mathrm{C}, 51.37 ; \mathrm{H}, 2.94 ; \mathrm{N}, 14.96$.

N'-(4-Cyanobenzylidene)-2-[(5-fluorobenzoxazol-2-yl)thio]acetohydrazide (3c)

M.p. $253.6^{\circ} \mathrm{C}$. Yield $90 \%$.

IR $v_{\max }\left(\mathrm{cm}^{-1}\right)$ : 3224.98 (N-H stretching), 3140.11, 3097.68, 3057.17 (Aromatic C-H stretching), 2978.09, 2941.44 (Aliphatic C-H stretching), 2229.71 ( $\mathrm{C} \equiv \mathrm{N}$ stretching), 1678.07 ( $\mathrm{C}=\mathrm{O}$ stretching), 1598.99, 1500.62, 1475.54, 1467.83 ( $\mathrm{N}-\mathrm{H}$ bending, $\mathrm{C}=\mathrm{N}$ and $\mathrm{C}=\mathrm{C}$ stretching), 1442.75, 1431.18, 1375.25 (C-H bending), 1346.31, 1309.67, 1219.01, 1201.65, 1130.29, 1105.21 (C-N, C-O stretching and aromatic C-H in plane bending), 954.76, 941.26, 881.47, 854.47, 844.82, 823.60, 785.03, 769.60, 677.01 (Aromatic C-H out of plane bending and C-S stretching).

${ }^{1} \mathrm{H}$ NMR (300 MHz, DMSO- $\left.d_{6}\right): 4.31$ and $4.70\left(2 \mathrm{~s}, 2 \mathrm{H}, \mathrm{S}-\mathrm{CH}_{2}\right), 7.15(\mathrm{t}, J=8.82 \mathrm{~Hz}, J=8.91 \mathrm{~Hz}, 1 \mathrm{H}, \mathrm{Ar}-\mathrm{H}), 7.50(\mathrm{~d}$, $J=8.31 \mathrm{~Hz}, 1 \mathrm{H}, \mathrm{Ar}-\mathrm{H}), 7.64-7.66(\mathrm{~m}, 1 \mathrm{H}, \mathrm{Ar}-\mathrm{H}), 7.88(\mathrm{~s}, 4 \mathrm{H}, \mathrm{Ar}-\mathrm{H}), 8.08$ and $8.27(2 \mathrm{~s}, 1 \mathrm{H}, \mathrm{CH}=\mathrm{N}), 12.03(\mathrm{~s}, 1 \mathrm{H}$, $\mathrm{N}-\mathrm{H})$. 
${ }^{13} \mathrm{C}$ NMR (75 MHz, DMSO-d $\left.d_{6}\right): 35.14\left(\mathrm{CH}_{2}, \mathrm{~S}-\mathrm{CH}_{2}\right), 105.46(\mathrm{CH}, \mathrm{d}, \mathrm{J}=26.25 \mathrm{~Hz}$, aromatic), $111.33(\mathrm{CH}, \mathrm{d}, J=9.75$ $\mathrm{Hz}$, aromatic), $111.87(\mathrm{CH}, \mathrm{d}, J=26.25 \mathrm{~Hz}$, aromatic), $112.33(\mathrm{C}$, aromatic), $119.10(\mathrm{C}, \mathrm{C} \equiv \mathrm{N}), 127.97(2 \mathrm{CH}$, aromatic), $133.16(2 \mathrm{CH}$, aromatic), 138.77 (C, aromatic), $142.68(\mathrm{C}$, aromatic), $145.83(\mathrm{CH}, \mathrm{CH}=\mathrm{N}), 148.28(\mathrm{C}$, aromatic), 158.31 (C, aromatic), 161.47 (C, aromatic), 168.70 (C, C=O).

HRMS (ESI) (m/z): [M+H] $]^{+}$calcd. for $\mathrm{C}_{17} \mathrm{H}_{11} \mathrm{FN}_{4} \mathrm{O}_{2} \mathrm{~S}: 355.0660$, found: 355.0643 .

For $\mathrm{C}_{17} \mathrm{H}_{11} \mathrm{FN}_{4} \mathrm{O}_{2} \mathrm{~S}$ Calculated: $\mathrm{C}, 57.62 ; \mathrm{H}, 3.13 ; \mathrm{N}, 15.81$. Found: $\mathrm{C}, 57.65 ; \mathrm{H}, 3.11 ; \mathrm{N}, 15.80$.

2-[(5-Fluorobenzoxazol-2-yl)thio]-N'-(4-fluorobenzylidene)acetohydrazide (3d)

M.p. $204.7^{\circ} \mathrm{C}$. Yield $84 \%$.

IR $v_{\max }\left(\mathrm{cm}^{-1}\right)$ : 3197.98 (N-H stretching), 3120.82, 3078.39, 3061.03 (Aromatic C-H stretching), 2980.02, 2960.73 (Aliphatic C-H stretching), 1674.21 (C=O stretching), 1616.35, 1600.92, 1498.69, 1479.40, 1469.76 (N-H bending, $\mathrm{C}=\mathrm{N}$ and $\mathrm{C}=\mathrm{C}$ stretching), 1435.04, 1381.03, 1357.89 (C-H bending), 1340.53, 1301.95, 1267.23, 1230.58, 1217.08, $1130.29,1103.28$ (C-N, C-O stretching and aromatic C-H in plane bending), 954.76, 931.62, 881.47, 860.25, $848.68,812.03,790.81,769.60,717.52,677.01$ (Aromatic C-H out of plane bending and C-S stretching).

${ }^{1} \mathrm{H}$ NMR (300 MHz, DMSO- $\left.d_{6}\right): 4.29$ and $4.67\left(2 \mathrm{~s}, 2 \mathrm{H}, \mathrm{S}-\mathrm{CH}_{2}\right), 7.14(\mathrm{t}, J=7.47 \mathrm{~Hz}, J=8.88 \mathrm{~Hz}, 1 \mathrm{H}, \mathrm{Ar}-\mathrm{H}), 7.26(\mathrm{t}$, $J=8.37 \mathrm{~Hz}, J=8.46 \mathrm{~Hz}, 2 \mathrm{H}, \mathrm{Ar}-\mathrm{H}), 7.49(\mathrm{~d}, J=8.46 \mathrm{~Hz}, 1 \mathrm{H}, \mathrm{Ar}-\mathrm{H}), 7.63-7.65(\mathrm{~m}, 1 \mathrm{H}, \mathrm{Ar}-\mathrm{H}), 7.76(\mathrm{~s}, 2 \mathrm{H}, \mathrm{Ar}-\mathrm{H})$, 8.04 and $8.22(2 \mathrm{~s}, 1 \mathrm{H}, \mathrm{CH}=\mathrm{N}), 11.80(\mathrm{~s}, 1 \mathrm{H}, \mathrm{N}-\mathrm{H})$.

${ }^{13} \mathrm{C}$ NMR (75 MHz, DMSO- $\left.d_{6}\right): 35.24\left(\mathrm{CH}_{2}, \mathrm{~S}-\mathrm{CH}_{2}\right), 105.42(\mathrm{CH}, \mathrm{d}, \mathrm{J}=26.25 \mathrm{~Hz}$, aromatic), $111.27(\mathrm{CH}, \mathrm{d}, \mathrm{J}=9.75$ $\mathrm{Hz}$, aromatic), $111.80(\mathrm{CH}, \mathrm{d}, \mathrm{J}=26.25 \mathrm{~Hz}$, aromatic), $116.31(2 \mathrm{CH}, \mathrm{d}, J=21.75 \mathrm{~Hz}$, aromatic), $129.56(\mathrm{CH}, \mathrm{d}, J=$ $8.25 \mathrm{~Hz}$, aromatic), $129.80(\mathrm{CH}, \mathrm{d}, \mathrm{J}=8.25 \mathrm{~Hz}$, aromatic), 130.93 (C, aromatic), 143.47 (C, aromatic), $146.66(\mathrm{CH}$, $\mathrm{CH}=\mathrm{N}), 148.28$ (C, aromatic), 158.30 (C, aromatic), 161.46 (C, aromatic), 165.17 (C, aromatic), $168.33(\mathrm{C}, \mathrm{C}=\mathrm{O})$.

HRMS (ESI) (m/z): $[\mathrm{M}+\mathrm{H}]^{+}$calcd. for $\mathrm{C}_{16} \mathrm{H}_{11} \mathrm{~F}_{2} \mathrm{~N}_{3} \mathrm{O}_{2} \mathrm{~S}: 348.0613$, found: 348.0597 .

For $\mathrm{C}_{16} \mathrm{H}_{11} \mathrm{~F}_{2} \mathrm{~N}_{3} \mathrm{O}_{2} \mathrm{~S}$ Calculated: $\mathrm{C}, 55.33 ; \mathrm{H}, 3.19 ; \mathrm{N}, 12.10$. Found: $\mathrm{C}, 55.35 ; \mathrm{H}, 3.21 ; \mathrm{N}, 12.07$.

N'-(4-Chlorobenzylidene)-2-[(5-fluorobenzoxazol-2-yl)thio]acetohydrazide (3e)

M.p. $208.9^{\circ} \mathrm{C}$. Yield $86 \%$.

IR $v_{\max }\left(\mathrm{cm}^{-1}\right)$ : 3230.77 (N-H stretching), 3138.18, 3076.46, 3057.17 (Aromatic C-H stretching), 2978.09 (Aliphatic C-H stretching), 1672.28 (C=O stretching), 1612.49, 1502.55, 1467.83 ( $\mathrm{N}-\mathrm{H}$ bending, $\mathrm{C}=\mathrm{N}$ and $\mathrm{C}=\mathrm{C}$ stretching), 1433.11, 1377.17, 1355.96 (C-H bending), 1338.60, 1303.88, 1219.01, 1203.58, 1128.36, 1103.28, 1087.85, 1014.56 (C-N, C-O stretching and aromatic C-H in plane bending), 952.84, 931.62, 881.47, 860.25, 839.03, 810.10, 783.10, 767.67, 713.66, 677.01 (Aromatic C-H out of plane bending and C-S stretching).

${ }^{1} \mathrm{H}$ NMR (300 MHz, DMSO- $\left.d_{6}\right): 4.28$ and $4.68\left(2 \mathrm{~s}, 2 \mathrm{H}, \mathrm{S}-\mathrm{CH}_{2}\right), 7.16(\mathrm{t}, J=8.97 \mathrm{~Hz}, J=9.06 \mathrm{~Hz}, 1 \mathrm{H}, \mathrm{Ar}-\mathrm{H}), 7.49(\mathrm{~d}$, $J=7.17 \mathrm{~Hz}, 3 \mathrm{H}, \mathrm{Ar}-\mathrm{H}), 7.65-7.68(\mathrm{~m}, 1 \mathrm{H}, \mathrm{Ar}-\mathrm{H}), 7.73(\mathrm{~d}, J=8.04 \mathrm{~Hz}, 2 \mathrm{H}, \mathrm{Ar}-\mathrm{H}), 8.03$ and $8.21(2 \mathrm{~s}, 1 \mathrm{H}, \mathrm{CH}=\mathrm{N})$, $11.86(\mathrm{~s}, 1 \mathrm{H}, \mathrm{N}-\mathrm{H})$.

${ }^{13} \mathrm{C}$ NMR (75 MHz, DMSO- $\left.d_{6}\right): 35.20\left(\mathrm{CH}_{2}, \mathrm{~S}-\mathrm{CH}_{2}\right), 105.46(\mathrm{CH}, \mathrm{d}, \mathrm{J}=26.25 \mathrm{~Hz}$, aromatic), 111.33 (CH, d, J= 10.5 $\mathrm{Hz}$, aromatic), $111.85(\mathrm{CH}, \mathrm{d}, \mathrm{J}=25.5 \mathrm{~Hz}$, aromatic), $129.04(2 \mathrm{CH}$, aromatic), $129.36(2 \mathrm{CH}$, aromatic), 133.29 (C, aromatic), 134.96 (C, aromatic), 143.33 (C, aromatic), $146.47(\mathrm{CH}, \mathrm{CH}=\mathrm{N}), 148.29(\mathrm{C}$, aromatic), $158.31(\mathrm{C}$, aromatic), 161.47 (C, aromatic), $168.40(\mathrm{C}, \mathrm{C}=\mathrm{O})$.

HRMS (ESI) $(\mathrm{m} / z):[\mathrm{M}+\mathrm{H}]^{+}$calcd. for $\mathrm{C}_{16} \mathrm{H}_{11} \mathrm{ClFN}_{3} \mathrm{O}_{2} \mathrm{~S}: 364.0317$, found: 364.0304 .

For $\mathrm{C}_{16} \mathrm{H}_{11} \mathrm{ClFN}_{3} \mathrm{O}_{2} \mathrm{~S}$ Calculated: $\mathrm{C}, 52.83 ; \mathrm{H}, 3.05 ; \mathrm{N}, 11.55$. Found: $\mathrm{C}, 52.79 ; \mathrm{H}, 3.06 ; \mathrm{N}, 11.58$. 
N'-(4-Bromobenzylidene)-2-[(5-fluorobenzoxazol-2-yl)thio]acetohydrazide (3f)

M.p. $194.4^{\circ} \mathrm{C}$. Yield $88 \%$.

IR $v_{\max }\left(\mathrm{cm}^{-1}\right)$ : 3246.20 (N-H stretching), 3142.04, 3076.46, 3055.24 (Aromatic C-H stretching), 2978.09 (Aliphatic C-H stretching), 1687.71 ( $\mathrm{C}=\mathrm{O}$ stretching), 1608.63, 1502.55, 1467.83 ( $\mathrm{N}-\mathrm{H}$ bending, $\mathrm{C}=\mathrm{N}$ and $\mathrm{C}=\mathrm{C}$ stretching), 1433.11, 1402.25, 1375.25, 1354.03 (C-H bending), 1336.67, 1303.88, 1217.08, 1203.58, 1126.43, 1101.35, 1070.49, 1010.70 (C-N, C-O stretching and aromatic C-H in plane bending), 952.84, 931.62, 881.47, 860.25, 835.18, 808.17, 781.17, 769.60, 707.88, 677.01 (Aromatic C-H out of plane bending and C-S stretching).

${ }^{1} \mathrm{H}$ NMR (300 MHz, DMSO-d $\left.d_{6}\right): 4.29$ and $4.67\left(2 \mathrm{~s}, 2 \mathrm{H}, \mathrm{S}-\mathrm{CH}_{2}\right), 7.14(\mathrm{t}, J=8.19 \mathrm{~Hz}, J=8.73 \mathrm{~Hz}, 1 \mathrm{H}, \mathrm{Ar}-\mathrm{H}), 7.49(\mathrm{~d}$, $J=8.43 \mathrm{~Hz}, 1 \mathrm{H}, \mathrm{Ar}-\mathrm{H}), 7.63-7.66(\mathrm{~m}, 5 \mathrm{H}, \mathrm{Ar}-\mathrm{H}), 8.01$ and $8.19(2 \mathrm{~s}, 1 \mathrm{H}, \mathrm{CH}=\mathrm{N}), 11.86(\mathrm{~s}, 1 \mathrm{H}, \mathrm{N}-\mathrm{H})$.

${ }^{13} \mathrm{C}$ NMR (75 MHz, DMSO- $\left.d_{6}\right): 35.23\left(\mathrm{CH}_{2}, \mathrm{~S}-\mathrm{CH}_{2}\right), 105.44(\mathrm{CH}, \mathrm{d}, J=26.25 \mathrm{~Hz}$, aromatic), $111.30(\mathrm{CH}, \mathrm{d}, J=9.75$ $\mathrm{Hz}$, aromatic), $111.83(\mathrm{CH}, \mathrm{d}, \mathrm{J}=26.25 \mathrm{~Hz}$, aromatic), 123.75 (C, aromatic), $129.24(2 \mathrm{CH}$, aromatic), $132.25(2 \mathrm{CH}$, aromatic), $133.61(\mathrm{C}$, aromatic), $143.43(\mathrm{C}$, aromatic), $146.56(\mathrm{CH}, \mathrm{CH}=\mathrm{N}), 148.28(\mathrm{C}$, aromatic), $158.30(\mathrm{C}$, aromatic), 161.46 (C, aromatic), $168.40(\mathrm{C}, \mathrm{C}=\mathrm{O})$.

HRMS (ESI) $(m / z):[\mathrm{M}+\mathrm{H}]^{+}$calcd. for $\mathrm{C}_{16} \mathrm{H}_{11} \mathrm{BrFN}_{3} \mathrm{O}_{2} \mathrm{~S}: 407.9812$, found: 407.9814 .

For $\mathrm{C}_{16} \mathrm{H}_{11} \mathrm{BrFN}_{3} \mathrm{O}_{2} \mathrm{~S}$ Calculated: $\mathrm{C}, 47.07 ; \mathrm{H}, 2.72 ; \mathrm{N}, 10.29$. Found: $\mathrm{C}, 47.07 ; \mathrm{H}, 2.70 ; \mathrm{N}, 10.31$.

$N^{\prime}$-([1,1'-Biphenyl]-4-ylmethylene)-2-[(5-fluorobenzoxazol-2-yl)thio]acetohydrazide (3g)

M.p. $215.8^{\circ} \mathrm{C}$. Yield $88 \%$.

IR $\mathrm{V}_{\max }\left(\mathrm{cm}^{-1}\right)$ : 3253.91 (N-H stretching), 3059.10, 3030.17 (Aromatic C-H stretching), 2927.94 (Aliphatic C-H stretching), $1658.78(\mathrm{C}=\mathrm{O}$ stretching), 1606.70, 1544.98, 1494.83, $1471.69(\mathrm{~N}-\mathrm{H}$ bending, $\mathrm{C}=\mathrm{N}$ and $\mathrm{C}=\mathrm{C}$ stretching), 1436.97, 1402.25, 1361.74 (C-H bending), 1311.59, 1238.30, 1193.94, 1136.07, 1130.29, 1064.71 (C-N, C-O stretching and aromatic C-H in plane bending), 991.41, 968.27, 956.69, 935.48, 887.26, 833.25, 813.96, 761.88, 736.81, 723.31, 713.66, 688.59, 675.09 (Aromatic C-H out of plane bending and C-S stretching).

${ }^{1} \mathrm{H}$ NMR (300 MHz, DMSO- $\left.d_{6}\right): 4.30$ and $4.71\left(2 \mathrm{~s}, 2 \mathrm{H}, \mathrm{S}-\mathrm{CH}_{2}\right)$, 7.14-7.20 (m, $\left.1 \mathrm{H}, \mathrm{Ar}-\mathrm{H}\right), 7.39-7.55(\mathrm{~m}, 4 \mathrm{H}, \mathrm{Ar}-\mathrm{H})$, 7.66-7.82 (m, 7H, Ar-H), 8.09 and $8.26(2 \mathrm{~s}, 1 \mathrm{H}, \mathrm{CH}=\mathrm{N}), 11.84(\mathrm{~s}, 1 \mathrm{H}, \mathrm{N}-\mathrm{H})$.

${ }^{13} \mathrm{C}$ NMR (75 MHz, DMSO- $\left.d_{6}\right): 35.29\left(\mathrm{CH}_{2}, \mathrm{~S}-\mathrm{CH}_{2}\right), 105.50(\mathrm{CH}, \mathrm{d}, \mathrm{J}=26.25 \mathrm{~Hz}$, aromatic), 111.37 (CH, d, J= 10.5 $\mathrm{Hz}$, aromatic), $111.88(\mathrm{CH}, \mathrm{d}, \mathrm{J}=26.25 \mathrm{~Hz}$, aromatic), $127.14(2 \mathrm{CH}$, aromatic), 127.51 (2CH, aromatic), 128.02 (2CH, aromatic), 128.38 ( $\mathrm{CH}$, aromatic), $129.50(2 \mathrm{CH}$, aromatic), 133.45 (C, aromatic), 139.75 (C, aromatic), $142.16(2 \mathrm{C}$, aromatic), $144.22(\mathrm{CH}, \mathrm{CH}=\mathrm{N}), 147.34(\mathrm{C}$, aromatic), 158.33 (C, aromatic), 161.48 (C, aromatic), $168.35(\mathrm{C}, \mathrm{C}=\mathrm{O})$.

HRMS (ESI) (m/z): [M+H] $]^{+}$calcd. for $\mathrm{C}_{22} \mathrm{H}_{16} \mathrm{FN}_{3} \mathrm{O}_{2} \mathrm{~S}: 406.1020$, found: 406.1010 .

For $\mathrm{C}_{22} \mathrm{H}_{16} \mathrm{FN}_{3} \mathrm{O}_{2} \mathrm{~S}$ Calculated: $\mathrm{C}, 65.17 ; \mathrm{H}, 3.98 ; \mathrm{N}, 10.36$. Found: $\mathrm{C}, 65.15 ; \mathrm{H}, 3.99 ; \mathrm{N}, 10.37$.

\subsection{Biochemistry}

\subsubsection{Cytotoxicity}

Cell culture and drug treatment

C6 Rat glioma and NIH/3T3 mouse embryonic fibroblast cells were incubated in Dulbecco's Modified Eagle's Medium (DMEM) (Sigma, Deisenhofen, Germany) supplemented with 10\% fetal calf serum (Gibco, Paisley, Scotland). All media were supplemented with $100 \mathrm{IU} / \mathrm{mL}$ penicillin-streptomycin (Gibco, Paisley, Scotland) and cells were incubated at $37^{\circ} \mathrm{C}$ in a humidified atmosphere of $95 \%$ air and $5 \% \mathrm{CO}_{2}$. Exponentially growing cells were plated at $2 \times 10^{4}$ cells $/ \mathrm{mL}$ into 96 -well microtiter tissue culture plates (Nunc, Denmark) and incubated for $24 \mathrm{~h}$ before the addition of the drugs (the optimum cell number for cytotoxicity assays was 
determined in preliminary experiments). The stock solutions of the compounds were prepared in dimethyl sulfoxide (DMSO; Sigma Aldrich, Poole, UK) and further dilutions were made with fresh culture medium (the concentration of DMSO in the final culture medium was $<0.1 \%$ which had no effect on the cell viability).

\section{MTT assay}

The level of cellular 3-(4,5-dimethylthiazol-2-yl)-2,5-diphenyltetrazolium bromide (MTT) (SigmaAldrich, St. Louis, MO, USA) reduction was quantified as previously described in the literature $[27,28]$ with small modifications [29].

After $24 \mathrm{~h}$ of preincubation, the compounds and mitoxantrone (positive control) were added to give final concentration in the range 3.9-500 $\mu \mathrm{g} / \mathrm{mL}$ and the cells were incubated for $24 \mathrm{~h}$. At the end of this period, MTT was added to a final concentration of $0.5 \mathrm{mg} / \mathrm{mL}$ and the cells were incubated for $4 \mathrm{~h}$ at $37^{\circ} \mathrm{C}$. After the medium was removed, the formazan crystals formed by MTT metabolism were solubilized by addition of 200 $\mu \mathrm{L}$ DMSO to each well and absorbance was read at $540 \mathrm{~nm}$ with a microtiter plate spectrophotometer (Bio-Tek plate reader, Winooski, VT, USA). Every concentration was repeated in three wells. IC $_{50}$ values were defined as the drug concentrations that reduced absorbance to $50 \%$ of control values.

Selectivity index (SI) values were calculated according to the formula [30] below:

$\mathrm{SI}=\mathrm{IC}_{50}$ for normal cell line $/ \mathrm{IC}_{50}$ for cancerous cell line

\subsubsection{Flow cytometric analyses of apoptosis}

After the cells were incubated with compound $3 \mathrm{~g}$ and mitoxantrone at $\mathrm{IC}_{50} / 2$ and $\mathrm{IC}_{50}$ concentrations, phosphatidylserine externalization, which indicates early apoptosis, was measured by FITC Annexin V apoptosis detection kit (BD Pharmingen, San Jose, CA, USA) on a BD FACSAria flow cytometer for $24 \mathrm{~h}$. Annexin V staining protocol was applied according to the manufacturer's instructions (BD Pharmingen, San Jose, CA, USA). The cells were then briefly washed with cold phosphate buffer saline (PBS) and suspended in a binding buffer at a concentration of $1 \times 10^{6}$ cells $/ \mathrm{mL}$. Then, $100 \mu \mathrm{L}$ of this solution containing $1 \times 10^{5}$ cells was transferred to a $5 \mathrm{~mL}$ test tube. After $5 \mu \mathrm{L}$ of Annexin V and PI was added, the cells were incubated for 15 min at room temperature in the dark. Then $400 \mu \mathrm{L}$ of $1 \mathrm{x}$ binding buffer was added to each tube and the cells were processed for data acquisition, and analyzed on a BD FACSAria flow cytometer using FACSDiva version 6.1.1 software (BD Biosciences, San Jose, CA, USA) [29].

\subsubsection{AChE inhibitory activity}

The AChE inhibitory effects of the compounds were determined by Ellman's method [31] with minor modifications (Electric eel AChE was used instead of bovine AChE and buffer was added $2.4 \mathrm{~mL}$ instead of 3 $\mathrm{mL}$ ) [32]. The compounds were dissolved in DMSO and tested at final concentration range from 5 to $80 \mu \mathrm{g} / \mathrm{mL}$. $20 \mu \mathrm{L}$ of $\mathrm{AChE}(1 \mathrm{U} / \mathrm{mL}), 10 \mu \mathrm{L}$ sample were added to $2.4 \mathrm{~mL}$ buffer, and the mixture was incubated at $37^{\circ} \mathrm{C}$ for $15 \mathrm{~min}$. After $15 \mathrm{~min}$ incubation, $50 \mu \mathrm{L}$ of $0.01 \mathrm{M}$ 5,5'-dithiobis(2-nitrobenzoic acid) (DTNB) and $20 \mu \mathrm{L}$ of $75 \mathrm{mM}$ acetylthiocholine iodide (ATCI) were added, and the final mixture was incubated at room temperature for $30 \mathrm{~min}$. Blank was prepared using $10 \mu \mathrm{L}$ of DMSO instead of the test sample, with all other procedures similar to those used in the case of the sample mixture. Absorbances were measured at $412 \mathrm{~nm}$ and $37^{\circ} \mathrm{C}$ using polystyrene cuvettes with spectrophotometer (UV-1700, Shimadzu). Experiment was done in triplicate. Galantamine was used as a positive control. Data are expressed as mean \pm standard deviation (SD). The inhibition (percent) of $\mathrm{AChE}$ was calculated using the following equation:

$$
\mathrm{I}(\%)=100-\left(\mathrm{OD}_{\text {sample }} / \mathrm{OD}_{\text {control }}\right) \times 100
$$

\subsection{In silico Prediction of ADME Parameters}

The physicochemical parameters (log P, TPSA, nrotb, molecular weight, number of hydrogen bond donors and acceptors, molecular volume) of the compounds were calculated using Molinspiration software [23-26]. 
Acknowledgements: This study was supported by Anadolu University Scientific Research Projects Commission under the grant no: $1705 S 175$.

Author contributions: Concept - M.D.A., G.A.Ç., H.E.T.; Design - M.D.A., G.A.Ç., H.E.T.; Supervision - M.D.A.; Resource - M.D.A., G.A.Ç., H.E.T.; Materials - M.D.A., G.A.Ç., H.E.T.; Data Collection and/or Processing - M.D.A., G.A.Ç., H.E.T.; Analysis and/or Interpretation - M.D.A.; Literature Search - M.D.A.; Writing - M.D.A.; Critical Reviews - M.D.A., G.A.Ç., H.E.T.

Conflict of interest statement: The authors declared no conflict of interest.

\section{REFERENCES}

[1] Lin L, Cai J, Jiang C. Recent Advances in Targeted Therapy for Glioma. Curr Med Chem. 2017; 24(13): 1365-1381. [CrossRef]

[2] Ghotme KA, Barreto GE, Echeverria V, Gonzalez J, Bustos RH, Sanchez M, Leszek J, Yarla NS, Gómez RM, Tarasov VV, Ashraf GM, Aliev G. Gliomas: New Perspectives in Diagnosis, Treatment and Prognosis. Curr Top Med Chem. 2017; 17(12): 1438-1447. [CrossRef]

[3] Patil SA, Hosni-Ahmed A, Jones TS, Patil R, Pfeffer LM, Miller DD. Novel approaches to glioma drug design and drug screening. Expert Opin Drug Discov. 2013; 8(9): 1135-1151. [CrossRef]

[4] Demmer CS, Bunch L. Benzoxazoles and oxazolopyridines in medicinal chemistry studies. Eur J Med Chem. 2015; 97: 778-785. [CrossRef]

[5] Rajasekhar S, Maiti B, Chanda K. A Decade Update on Benzoxazoles, a Privileged Scaffold in Synthetic Organic Chemistry. Synlett 2017; 28: 521-541. [CrossRef]

[6] Rida SM, Ashour FA, El-Hawash SAM, ElSemary MM, Badr MH, Shalaby MA. Synthesis of some novel benzoxazole derivatives as anticancer, anti-HIV-1 and antimicrobial agents. Eur J Med Chem. 2005; 40(9): 949-959. [CrossRef]

[7] Abdelgawad MA, Belal A, Omar HA, Hegazy L, Rateb ME. Synthesis, anti-breast cancer activity, and molecular modeling of some benzothiazole and benzoxazole derivatives. Arch Pharm Chem Life Sci. 2013; 346(7): 534-541. [CrossRef]

[8] An Y, Lee E, Yu Y, Yun J, Lee MY, Kang JS, Kim W-Y, Jeon R. Design and synthesis of novel benzoxazole analogs as Aurora B kinase inhibitors. Bioorg Med Chem Lett. 2016; 26(13): 3067-3072. [CrossRef]

[9] Abdelgawad MA, Bakr RB, Omar HA. Design, synthesis and biological evaluation of some novel benzothiazole/benzoxazole and/or benzimidazole derivatives incorporating a pyrazole scaffold as antiproliferative agents. Bioorg Chem. 2017; 74: 82-90. [CrossRef]

[10] Belal A, Abdelgawad MA. New benzothiazole/benzoxazole-pyrazole hybrids with potential as COX inhibitors: design, synthesis and anticancer activity evaluation. Res Chem Intermed. 2017; 43(7): 3859-3872. [CrossRef]

[11] Taskin T, Yilmaz S, Yildiz I, Yalcin I, Aki E. Insight into eukaryotic topoisomerase II-inhibiting fused heterocyclic compounds in human cancer cell lines by molecular docking. SAR QSAR Environ Res. 2012; 23: 345-355. [CrossRef]

[12] Yaghmaei S, Ghalayania P, Salami S, Nourmohammadian F, Koohestanimobarhan S, Imeni V. Hybrid benzoxazolecoumarin compounds induce death receptor-mediated switchable apoptotic and necroptotic cell death on HN-5 head and neck cancer cell line. Anti-Cancer Agents Med Chem. 2017; 17(4): 608-614. [CrossRef]

[13] Mathew B, Suresh J, Ahsan MJ, Mathew GE, Usman D, Subramanyan PNS, Safna KF, Maddela S. Hydrazones as a privileged structural linker in antitubercular agents: A Review. Infect Disord Drug Targets. 2015; 15(2): 76-88. [CrossRef]

[14] Narang R, Narasimhan B, Sharma S. A review on biological activities and chemical synthesis of hydrazide derivatives. Curr Med Chem. 2012; 19(4): 569-612. [CrossRef]

[15] Kumar P, Narasimhan B. Hydrazides/hydrazones as antimicrobial and anticancer agents in the new millennium. Mini-Rev Med Chem. 2013; 13(7): 971-987. [CrossRef]

[16] Vogel S, Kaufmann D, Pojarová M, Müller C, Pfaller T, Kühne S, Bednarski PJ, von Angerer E. Aroyl hydrazones of 2-phenylindole-3-carbaldehydes as novel antimitotic agents. Bioorg Med Chem. 2008; 16(12): 6436-6447. [CrossRef]

[17] Xia Y, Fan C-D, Zhao B-X, Zhao J, Shin D-S, Miao J-Y. Synthesis and structure-activity relationships of novel 1arylmethyl-3-aryl-1H-pyrazole-5-carbohydrazide hydrazone derivatives as potential agents against A549 lung cancer cells. Eur J Med Chem. 2008; 43(11): 2347-2353. [CrossRef] 
[18] Alam MS, Lee D-U. Synthesis, biological evaluation, drug-likeness, and in silico screening of novel benzylidenehydrazone analogues as small molecule anticancer agents. Arch Pharm Res. 2016; 39(2): 191-201. [CrossRef]

[19] Şenkardeş S, Kaushik-Basu N, Durmaz İ, Manvar D, Basu A, Atalay R, Küçükgüzel ŞG. Synthesis of novel diflunisal hydrazide-hydrazones as anti-hepatitis $C$ virus agents and hepatocellular carcinoma inhibitors. Eur J Med Chem. 2016; 108: 301-308. [CrossRef]

[20] Alam MS, Choi S-U, Lee D-U. Synthesis, anticancer, and docking studies of salicyl-hydrazone analogues: A novel series of small potent tropomyosin receptor kinase A inhibitors. Bioorg Med Chem. 2017; 25(1): 389-396. [CrossRef]

[21] Despaigne AAR, Vieira LF, Mendes IC, da Costa FB, Speziali NL, Beraldo H. Organotin(IV) complexes with 2acetylpyridine benzoyl hydrazones: Antimicrobial activity. J Braz Chem Soc. 2010; 21: 1247-1257. [CrossRef]

[22] Altıntop MD, Özdemir A, Turan-Zitouni G, Ilgın S, Atlı Ö, İşcan G, Kaplancıklı ZA. Synthesis and biological evaluation of some hydrazone derivatives as new anticandidal and anticancer agents. Eur J Med Chem. 2012; 58: 299_ 307. [CrossRef]

[23] Molinspiration Cheminformatics. http://www.molinspiration.com. (accessed December 17, 2017).

[24] Lipinski CA, Lombardo F, Dominy BW, Feeney PJ. Experimental and computational approaches to estimate solubility and permeability in drug discovery and development settings. Adv Drug Deliv Rev. 2001; 46: 3-26. [CrossRef]

[25] Veber DF, Johnson SR, Cheng H-Y, Smith BR, Ward KW, Kopple KD. Molecular properties that influence the oral bioavailability of drug candidates. J Med Chem. 2002; 45(12): 2615-2623. [CrossRef]

[26] Gabr MT, El-Gohary NS, El-Bendary ER, El-Kerdawy MM, Ni N. Synthesis, in vitro antitumor activity and molecular modeling studies of a new series of benzothiazole Schiff bases. Chinese Chem Lett. 2016; 27(3): 380-386. [CrossRef]

[27] Mosmann T. Rapid colorimetric assay for cellular growth and survival: application to proliferation and cytotoxicity assays. J Immunol Methods. 1983; 65: 55-63. [CrossRef]

[28] Keiser K, Johnson CC, Tipton DA. Cytotoxicity of mineral trioxide aggregate using human periodontal ligament fibroblasts. J Endod. 2000; 26(5): 288-291. [CrossRef]

[29] Altıntop MD, Temel HE, Sever B, Akalın Çiftçi G, Kaplancıklı ZA. Synthesis and evaluation of new benzodioxolebased thiosemicarbazone derivatives as potential antitumor agents. Molecules 2016; 21(11), 1598. [CrossRef]

[30] Zhou HY, Dong FQ, Du XL, Zhou ZK, Huo HR, Wang WH, Zhan HD, Dai YF, Jing Meng, Sui YP, Li J, Sui F, Zhai YH. Antitumor activities of biscoumarin and dihydropyran derivatives. Bioorg Med Chem Lett. 2016; 26(16): 38763880. [CrossRef]

[31] Ellman GL, Courtney KD, Andres V, Featherstone RM. A new and rapid colorimetric determination of acetylcholinesterase activity. Biochem Pharmacol. 1961; 7(2): 88-95. [CrossRef]

[32] Turan-Zitouni G, Altıntop MD, Özdemir A, Kaplancıklı ZA, Akalın Çiftçi G, Temel HE. Synthesis and evaluation of bis-thiazole derivatives as new anticancer agents. Eur J Med Chem. 2016; 107: 288-294. [CrossRef] 